\title{
Beyond the Rhetoric: An Introduction to Implementing TELRIC
}

\author{
D. MARK KENNET* \\ LECOM Associates Inc. \\ and \\ RAÚL PEREZ-REYES
}

Organismo Supervisor para la Inversión Privada en Telecomunicaciones

\begin{abstract}
This paper demonstrates the feasibility of the TELRIC standard. We develop an engineering-economic model of an interoffice telephone network suitable for calculating a TELRIC interconnection cost. The model utilizes the method of simulated annealing to determine an "optimal" SONET ring. Using actual switch location data for Maryland we calculate such a network and demonstrate its incremental cost for interconnection.
\end{abstract}

\section{Introduction}

On 13 May 2002, in Verizon et al. v. FCC et al. (2002) the United States Supreme Court essentially overturned a previous decision by the Eighth Circuit Court in which the latter had ruled against the FCC's adoption of a new cost measurement standard, called TELRIC (for "Total Element Long Run Incremental Cost"). In this paper, we do not attempt to review the legal arguments or attempt to divine the intentions of parties that opposed the FCC standard. We also do not wish to revisit the arguments over whether cost-based prices of any sort comport with various notions of economic efficiency. Instead, we attempt to demonstrate the feasibility of implementing the TELRIC standard and argue that its application is no more theoretical than any other cost measurement approach, including accounting cost.

The rest of the paper is organized as follows. In the next section, we introduce the concept of TELRIC. In section 3, we present a bottom-up model of inter-exchange trunking suitable for TELRIC computations. Section 4 discusses the various types of data needed to calculate costs, and section 5 presents some results for publicly available data from the state of Maryland. Finally, in section 6 we discuss some tentative conclusions.

\footnotetext{
* Contact author. Mailing address: Senior Economic Advisor, Organismo Supervisor para la Inversión Privada en Telecomunicaciones, La Prosa 136, San Borja, Lima, Peru, Ph 5112251313 anexo 558. E-mail: MarkKennet@hotmail.com.
} 


\section{TELRIC: An application to interconnection costs}

The FCC (1996) indicated that it was suggesting to state regulators in the U.S. that they implement cost-based tariffs for interconnection and for unbundled network elements using a methodology defined in the Order. This methodology, which the FCC termed "Total Element Long Run Incremental Cost," intended to avoid a number of problems encountered by regulators with other costing approaches, by calculating the incremental cost of elements used to create services rather than the cost of the services themselves.

In order to accomplish the calculation, it is necessary to have a model that permits examination of those components of the network utilized to provide the service. Put another way, it is necessary to have a model that is sufficiently disaggregated that it can examine individual network components as they are configured to provide desired services efficiently.

For example, the incremental cost of interconnection is treated as the average incremental cost of the elements - switch and link capacity - required to manage the extra traffic associated with an interconnecting network. By pricing elements, the standard promises to explicitly award a portion of common cost to each service, offering the incumbent a return on its investment to the extent the firm has invested efficiently.

However, while the FCC's approach offers a number of advantages, there are several disadvantages including the fact that here is no generally accepted methodology for performing TELRIC calculations. ${ }^{1}$ The FCC has provided some guidance through the release of its HCPM software, a variant of which is used to perform the calculations in this paper.

Some authors (for example, DeGraba (2000)) have argued that because it is not feasible to calculate costs, and given the flow of benefits arising from interconnection externalities, that a "bill and keep" regime should be employed wherein each interconnection company would simply charge its own users for its perceived cost of interconnection and the government need intervene no further. We argue that if one is willing to accept the notion of a "standard" network architecture for the purpose of calculating long run costs, it is actually quite feasible to calculate realistic costs of interconnection. In the next section, we describe the features of one such standardized model.

\section{A model for calculating interconnection costs}

\subsection{Model underpinnings}

The basic objective of any TELRIC model is to simulate the construction of a telephone network using the principles of efficient design both technologically and economically. This approach to modelling is in contrast to more traditional accounting methods, which in

\footnotetext{
${ }^{1}$ One of the more controversial issues, especially in developing countries, is related to the technology used in transmission modeling. In Peru, for example, in the mountains it is very difficult to install optical fiber because of river overflow. In that case it is necessary to use microwave or satellite, even though it might appear that in terms of minimizing cost these technologies are not correct. The TELRIC approach requires a degree of variety, in order to capture certain aspects of the real world. In the modeling of switching, the problems are not less than in transmission modeling. Both cases require a hands-on approach; these particularities make the differences in TELRIC models.
} 
addition to suffering the problems observed in recent days with respect to Enron, Global Crossing, MCI Worldcom, Vivendi, etc., do not offer the possibility of adequately exploring in detail the disaggregated network. Essentially, one can think of a TELRIC model as a simulation analysis, wherein a network is "constructed" to meet current demand levels and quality of service using actual customer and switch locations. ${ }^{2}{ }^{3}$ The FCC's outside plant model meets this standard by simulating the plant necessary between switch and customer locations given any level of service quality; this portion of the HCPM software is being used in the determination of universal service subsidies in the United States and has been described in Bush et al. (2001). In this paper we describe the HCPM module designed to calculate the cost of the telephone system plant that carries traffic between switching offices. ${ }^{4}$

The interoffice computations in HCPM are accomplished in two main modules, which are further divided into sub-modules. The interoffice cluster module uses cluster analysis to group switches into a hierarchical system of rings in order to provide full connectivity in the interoffice network. In the interoffice design module which follows this module, the actual interoffice routes are computed and the investment in structures, fiber and terminal capacity is determined. In contrast to the architecture of the local loop, which is based on a tree design, the interoffice connections are commonly made by ring topologies. While not as efficient as a tree in establishing basic connectivity, a ring has the advantage that there are two paths between every pair of nodes, depending on the direction in which messages are transmitted around the ring. Thus, if any single link on the ring is disabled, full connectivity between every pair of nodes can still be maintained.

The remainder of this section describes the engineering assumptions and algorithms used to calculate interoffice and switching costs, and then describes methodology which may be used to allocate cost of shared components to individual wire centers.

\subsection{Interoffice clustering module (IOCLUST)}

The interoffice clustering module, or IOCLUST, is designed to allow the user a range of options in designing the interoffice network, based on the locations of switching centers. An input file $<$ areaname.csv $>$ provides the basic data which this module requires. This file, which is a text file in ".csv" format lists in the identification code for all of the switches in the serving area followed by data for each switch in fields separated by commas. In the second field (column 2) the serving tandem switch is identified. There are several options for identifying tandem switches that will be described in detail later. The remaining fields in the input data file describe the longitude and latitude of the switch, the number of access lines connected to the switch, the total peak hour usage of the switch measured in terms of the North American unit of CCS (hundred call seconds), peak hour interoffice usage

\footnotetext{
2 The FCC apparently concluded that it was necessary to hold existing switch locations fixed in TELRIC computations. One can easily imagine that taking the original logic seriously implies what has become known in the jargon as a "scorched earth" model wherein a network is designed taking no network elements as given. ${ }^{3}$ In some countries (for example, Peru), the legal framework establishes that the regulator develop a parallel model to the one developed by the firms, and the final decision mandating, e.g., an interconnection tariff, reconciles both models. This is intended as a way to reduce the regulatory asymmetry while assuring model realism.

4 Another TELRIC model, LECOM, is described in Gasmi et al. (2002), and is applied extensively in that book to address a number of issues in economic and regulation theory.
} 
measured in CCS, and the total annual minutes of use (in peak and off-peak periods). The final six fields contain additional data on the line side connections into the switch, including the number of business DS0 lines, business DS1 lines, residential DS0 lines, residential DS1 lines, the number of DLCs and the number of special access lines. This information can be automatically updated each time that the loop design module of the HCPM (feeddist.exe) is run.

IOCLUST can be run in various modes. When run using a command line option specifying the area name corresponding to the .csv input file, the module is started by inserting in a batch file or other program the instructions "IOClust.exe <areaname>". For example, to run the model for the state of Idaho using the input file Idaho.csv, the user would type "IOClust idaho". Alternatively, by entering only the module name "IOClust" the module is started and the user has the option of entering the areaname and modifying any of the user adjustable parameters before running the module. Finally, IOCLUST is invoked automatically from the INTEROFFICE user interface when a complete model run is being executed.

After reading in the input data file, the interoffice clustering module determines whether or not a set of tandem switches has been defined in the input file. It is possible to identify a set of tandem switches simply by repeating the text string in field 1, which identifies the switch, in field 2 under the "tandem" heading. It is also possible to assign any number of additional switches to a particular tandem, by placing the location identifier for the appropriate tandem in field 2. If no tandem switches are defined in the user input file, then a set of tandems will be determined by the interoffice cluster module where the number of tandem switches is determined by cluster analysis using some user specified engineering principles which specify the maximum number of host switches on an interoffice ring and/or the minimum number of tandem zones which should be created. For example, if the minimum number of zones selected is equal to 1 , then the number of rings is completely determined by the nodes per ring constraint. On the other hand, if the maximum number of nodes per ring is specified as any number greater than the total number of host switches, then the number of rings is completely determined by the minimum number of zones constraint. These two constraints can be used in combination, so that either one, or both of the constraints can be binding for any particular study area.

After making an assignment of switches to tandems, the interoffice clustering module creates configurations of host and remote switches. These computations are based on cluster analysis methods, and are constrained by user selected input values which determine the maximum number of lines in a remote switch and the maximum number of lines in a host-remote ring. Each of the user input values can be specified in the input file "IOClust.txt" and these values are read by the program each time that it is called.

Outputs of the interoffice cluster module, which are passed as inputs to the interoffice design module are contained in the files: "remotei.csv", "hosti.csv" and "tandem.csv." These files contain, respectively, a list of remote switches associated with tandem " $i$ ", the host switches associated with tandem "i" and the set of tandem switches.

\subsection{A model of interoffice plant}

The HCPM interoffice module borrows much of its basic engineering design methodology from the earlier Benchmark Cost Proxy Model, written by INDETEC International, Inc., and the HAI Model, written by HAI, Inc. Essentially, the module simulates a SONET ring 
network interconnecting all switches in a pre-determined region, along with any associated remote and tandem switches. ${ }^{5}$ The user may define these groupings independently or use the included IOCLUST module to attempt to model the most efficient configuration. The tandem switch associated with each SONET ring is in turn interconnected with other tandems via another SONET ring structure.

SONET link capacity is determined by calculating the total peak load traffic for a given ring and using either the Poisson or Erlang-B (a user option) probability distribution to determine the size of links between offices. The derivation of the Poisson formula is as follows. Suppose that calls arrive at a Poisson rate $\lambda$ (peak usage in Erlangs), and there are $\mathrm{N}$ channels available. Then the probability that the number of calls $\mathrm{n}$ equals the number of channels $\mathrm{N}$ is

$$
\operatorname{Pr}\{n=N\}=e^{-\lambda} \lambda^{N} / N !
$$

Then the probability of a call being blocked is equal to the probability that $\mathrm{N}$ or more channels have already been occupied, or $\operatorname{Pr}\{n \geq N\}=1-\operatorname{Pr}\{n<N\}$ :

$$
\operatorname{Pr}\{n \geq N\}=1-e^{-\lambda} \sum_{j=0}^{N-1} \frac{\lambda^{j}}{j !}
$$

For any peak usage level, this equation can be solved iteratively for the number of channels that gives a blocking probability less than or equal to the desired level.

The Erlang-B blocking probability is given by

$$
B(\lambda, N)=\frac{\frac{\lambda^{N}}{N !}}{\sum_{j=0}^{N} \frac{\lambda^{j}}{j !}}
$$

which can also be iteratively solved for $\mathrm{N}$.

The installed cost of each link in the ring depends on both the capacity of the link (constant throughout the ring) and the distance. Thus, analogously to the case of FEEDDIST, we account for both cable and structure costs. One could also introduce variation in these costs based on either line density or terrain type; we will abstract from these features.

For each type of investment, the model assumes that an annual charge factor can be calculated (and is input by user-determined parameters). The annual charge factor is the

\footnotetext{
5 The existence of a system of transmission based on SONET permits a homogenous communications network, with standardized manageable interfaces. Given the current state of technological advance, SONET represents the network of future transport for the services of broadband, based on technologies like ATM.
} 
ratio of annual expenses to the total investment in the SONET ring and includes cost of capital, operation expenses, maintenance, and depreciation.

The routing of each SONET ring is optimized for minimum structure cost. The optimization routine makes use of the fact that connecting points on a map is very similar to the classical "traveling salesman problem." We note that while this problem belongs to the class of problems known in the computer science literature as NP-complete (i.e., it is impossible to guarantee a global solution in a number of iterations expressible as a function of the number of points to be connected), there are several good algorithms available that approximate the global solution. We employ one of these approximate approaches, the Metropolitan et al. simulated annealing algorithm described in Press, et al. (1986).

Essentially, the simulated annealing algorithm employs the principle that the optimal solution may not lie on a direct path from the starting point in the direction of maximum descent. Much like using a torch to heat cast iron objects while they cool (annealing), the algorithm randomly chooses an occasional search direction different from maximum descent. For more specific details, consult Press, et al. (1986).

\subsection{A model of local switching}

The local switch module is based upon the design principles of the Northern Telecom DMS-100 host-remote system, but is intended to be a generic model of a modern switch. Switching cost is determined by the number of DS0 lines, DS1 lines, and peak hourly usage for the whole office and interoffice (interoffice traffic must be less than or equal to the total for the switch). In the case of the host switch, the number of attached remotes and their features must also be taken into account. All lines are considered in terms of DS0equivalents.

First, the model considers the number of switch co-locations. If the total number of lines existing at a switch location exceeds the line capacity of the switch (a user input), the appropriate number of replicates of the switch are designed to be collocated. If the total number of lines is zero, then only the getting started cost of the switch is calculated.

The next section of code takes care of the cost of terminating digital lines on the host switch. Subscriber carrier modules are assumed to connect directly to the network plane, with the number of ports per module a user input.

Type B line cards, which are used for party lines, ground start $\mathrm{PBX}^{6}$, and coin-operated public telephones are incorporated into the model. The model assumes that no party lines exist; coin-operated public telephones are based on an assumed (user-input) percentage of switched lines. All remaining lines are served by Type A line cards.

Each line card is fitted in a line concentrating drawer; the capacity of each drawer is a user input. Drawers, in turn, are fitted into line concentrating modules, and modules are fitted into frames; again, the number of drawers per module and the number of modules per frame are determined by the user.

Links from concentrating modules to ports are determined from a table based on Poisson or Erlang-B values. Links between the host and remote are calculated similarly.

The number of ports is used to calculate the number of line group controllers, which are fitted to a line group equipment frame. The line group controllers interface with the line modules. Digital trunk controllers are fitted to digital trunk equipment frames.

\footnotetext{
${ }^{6}$ A ground-start PBX is a private branch exchange (PBX) that uses a ground in place of a second wire for completing the circuit between the PBX and the central office.
} 
The switch network is assumed to be an enet, with virtually unlimited capacity. Provision is also made for general costs, such as power, small items related to number of lines and/or usage, call-related, and trunk-related costs. The cost of the main distribution frame is calculated as a fixed cost plus a cost per switched line.

Each of the components described in the paragraph above is accounted for within the module. Costs are determined by the user, as are all of the capacities.

Finally, as in the other modules, a percentage of NTS investment (determined by the user) is assumed to be added onto equipment investment for building and land associated with that type of capital. Annual charge factors associated with circuit equipment, buildings, and land are applied to the respective investments for the calculation of annual cost as in other portions of the model.

\subsection{Incremental cost methodology.}

The output report from the HCPM Interoffice module is designed to facilitate various incremental cost calculations, including TSLRIC and TELRIC, without explicitly doing the calculations per se. ${ }^{7}$ Thus, the following costs (both investments and expenses) are reported for each interoffice network ring (tandem, host, and remote).

- $\quad$ Total ring network cost, including leased lines

- Total ring cost, with no traffic and no leased lines

- Total ring cost, switched lines only

- $\quad$ Total ring cost, leased lines only

In addition, the incremental ring cost is reported in turn for each office in the ring, with that office removed from the network. That is, suppose a network consisted of nodes A, B, and $\mathrm{C}$. Then the incremental cost of node $\mathrm{A}$ is simply the total cost of the network ABC less the cost of network $\mathrm{BC}$, removing all lines and traffic associated with node A.

The incremental cost calculation at each node can be used if a fully disaggregated interconnection cost calculation is desired rather than an average system-level cost. There might be several possible applications. If the intention is to design a purely per-line or a two-part interconnection tariff, the incremental cost of each node can be used as the basis for the per-line rate at that node, with the per-minute rate based on an incremental cost calculation for traffic.

Suppose that the cost of interconnection for a "foreign" network $\mathrm{X}$ at a point $\mathrm{A}$ in a network $\mathrm{ABC}$ is desired. A's incremental contribution to the cost of network $\mathrm{ABC}$ is known from the calculation above, and we would be interested in knowing what to allocate to $\mathrm{X}$. This can be obtained by calculating the cost of the network $(\mathrm{A}+\mathrm{X}) \mathrm{BC}$ and isolating the incremental cost of $\mathrm{A}+\mathrm{X}$. The difference between the incremental cost of $\mathrm{A}+\mathrm{X}$ and $\mathrm{A}$ is the total incremental cost of X, which includes X's traffic. We can now use the incremental cost of X's traffic as calculated in the manner described earlier to allocate the total incremental cost of $\mathrm{X}$ between per-line and per-minute costs in the following identity:

\footnotetext{
${ }^{7}$ One could argue that the choice of incremental cost methodology is a policy decision that is appropriately made outside the costing model.
} 
$\operatorname{TIC}(X)=\operatorname{IncrementalTrafficCost}(X) / \operatorname{AnnMin}(X) * \operatorname{AnnMin}(X)+\operatorname{PerLineCost}(X) * \operatorname{Lines}(X)$

In this identity, $\mathrm{TIC}(\mathrm{X})$ and IncrementalTrafficCost $(\mathrm{X})$ are known as above, and $\operatorname{AnnMin}(\mathrm{X})$ and Lines(X) are given. Thus, we can solve for PerLineCost $(\mathrm{X})$.

Incremental costs in switching are determined in a manner similar to that of interoffice trunks. First, switching cost is calculated using the demand present at each switch. Next, cost is calculated setting peak traffic to zero. Switch cost when peak traffic is zero is equal to non-traffic-sensitive cost (NTS), and the difference between the two calculated costs is equal to TS cost.

\section{Data}

The program uses the following demand elements in its calculations. These data are entered in an Excel spreadsheet and saved in comma-separated variable (CSV) format.

CLLI: Switch identifier.

Tandem: The serving tandem switch

Lon: Longitude of the switch

Lat: Latitude of the switch

Lines: The number of access lines connected to the switch

TotCCS: Total busy-hour traffic in hundreds of calling seconds (CCS) for the node. In the case of total ring calculations, a coincident peak is assumed and totals for all nodes in the ring are reported. Note that $36 \mathrm{CCS}=1$ erlang.

IOCCS: Total busy-hour interoffice CCS for the node. In the case of total ring calculations and for tandems, a coincident peak is assumed and totals for all nodes in the ring are reported.

AnnMin: Total annual minutes of traffic for the node. Values for total ring calculations are the sum of component node traffic plus the percentage of traffic that goes off-ring (a user input).

BUSDS0: Total business single-channel lines

BusDS1: Total business digital lines, in single-channel equivalents.

ResDS0: Total residential single-channel lines.

ResDS1: Total residential digital lines, in single-channel equivalents.

DLCs: The number of DLCs 
SpclAcc: The number of special access lines

In addition, the program requires information on a fairly large number of network elements. These data and default values are available from the authors, as is the program and source code listing.

\section{$5 \quad$ Results}

We have calculated the cost of a simulated interoffice network for the state of Maryland using publicly available data on locations of central offices and tandems. Figure 1 shows a graph of the simulated interoffice network, which consists of three interconnected SONET rings (each central office is assigned to a ring which contains one tandem switch; there are three tandem switches in the state).

In Figure 1, the green ring represents the tandem ring, red rings are host rings, and blue rings are remote rings. Executing the program results in an estimated total interoffice annual cost of about $\$ 222$ million, including the cost of local switching. Table 1 gives summary results. Note that expenses per line are annual expenses per line; these should be divided by 12 to reflect what a consumer would see on a phone bill. Thus, the average consumer's share of the fixed cost of switching according to these results is about $\$ 2$ per month.

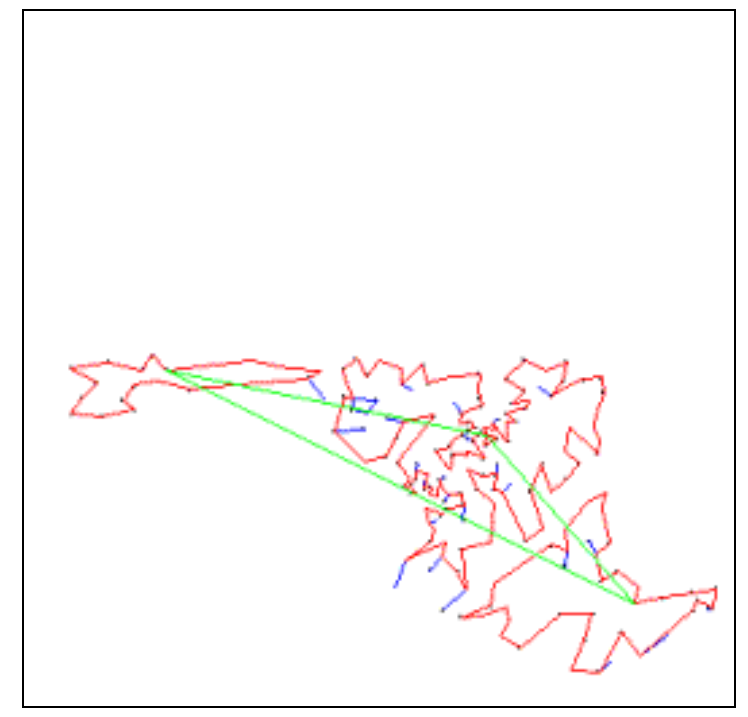

Figure 1

In the demand data, we have assumed that the average telephone line is utilized approximately 15 minutes per day, a fairly conservative estimate. Dividing all results by total annual usage results in an average expense per minute of approximately one-half cent. Note, however, that this result is highly sensitive to usage, and if actual usage were to double without changing peak demand, this expense would be reduced by one-half. We further note that nearly all of this expense occurs in switching; annual expense per line attributable to interoffice links is practically insignificant. 


\begin{tabular}{|l|l|l|l|}
\hline & Switching & Trunks & Total \\
\hline Total NTS Expense & $\$ 178,555,043.54$ & $\$ 2,572,523.91$ & $\$ 181,127,567.45$ \\
\hline Total TS Expense & $\$ 12,730,343.61$ & $\$ 27,938,161.36$ & $\$ 40,668,504.97$ \\
\hline Total Expense & & $\$ 221,796,072.42$ \\
\hline Total Lines & & $7,299,306$ \\
\hline Total Ann Min & & $40,843,083,492$ \\
& & \\
\hline NTS Expense per line & $\$ 24.46$ & $\$ 0.35$ & $\$ 24.81$ \\
\hline TS Expense per minute & $\$ 0.0003$ & $\$ 0.0007$ & $\$ 0.0010$ \\
\hline & & $\$ 0.0054$ \\
\hline Total expense per minute & $\$ 0.0047$ & $\$ 0.0007$ & \\
\hline & & 5595.474897 \\
\hline Annual minutes per line & & 466.2895748 \\
\hline Monthly minutes per line & & 15.33006821 \\
\hline Daily minutes per line & & \\
\hline
\end{tabular}

Table 1: Annual interconnection costs for Maryland wire centers

Using this line of reasoning suggests immediately that any price for interconnection ought to be a charge based on the system capacity utilized by the interconnecting firm; i.e., a price per line plus some cost for any change in peak capacity required as a result of the interconnection. Such a charge could take the form of a simple linear per-line charge, or a two-part tariff involving a charge per line plus a charge per CCS (or Erlang). In this paper, however, we do not advocate a pricing scheme but merely seek to investigate the costs of interconnection.

To calculate such a cost, we need to define some increment to the system described above and calculate the cost differential. Suppose an interconnecting firm were responsible for driving up peak usage by $3600 \mathrm{CCS}$, or 100 Erlangs. $^{8} \mathrm{We}$ alter the demand database for Maryland to reflect this increase in peak demand, and re-run the model to obtain the results in Table 2.

We note that all differences between costs calculated in Tables 2 and 1 occur in the traffic sensitive categories, resulting in a total incremental cost of $\$ 5,094,675.83$, which, when divided by annual minutes, results in an incremental cost of 0.02 cents per minute. Once again, we point out that this result depends critically on the number of minutes that are used as the denominator, and that these minutes do not drive the total cost of the system.

As we suggest earlier, it may be more economically satisfying to determine costs per unit of capacity, since it is capacity that drives total cost in the first place. Using the results from the model, we find that in the original run, annual costs were \$25.91 per CCS, while

\footnotetext{
${ }^{8} \mathrm{CCS}$ is the number (in hundreds) of calling seconds during the peak hour of utilization. An Erlang is the capacity of a fully utilized channel. That is, one line completely occupied for one hour equals 1 Erlang. Thus, $36 \mathrm{CCS}=1$ Erlang.
} 
with the increased usage in the second run costs fell to $\$ 24.35$ per CCS. The incremental cost per CCS was $\$ 6.71$, or $\$ 241.56$ per Erlang.

\begin{tabular}{|c|c|c|c|}
\hline & Switching & Trunks & Total \\
\hline Total NTS Expense & $\$ 178,555,043.54$ & $\$ 2,572,523.91$ & $\$ 181,127,567.45$ \\
\hline Total TS Expense & $\$ 16,033,536.42$ & $\$ 29,729,644.38$ & $\$ 45,763,180.80$ \\
\hline Total Expense & & & $\$ 226,890,748.25$ \\
\hline Total Lines & & & $7,299,306$ \\
\hline Total Ann Min & & & $40,843,083,492$ \\
\hline NTS Expense per line & $\$ 24.46$ & $\$ 0.35$ & $\$ 24.81$ \\
\hline TS Expense per minute & $\$ 0.0004$ & $\$ 0.0007$ & $\$ 0.0011$ \\
\hline Total expense per minute & $\$ 0.0048$ & $\$ 0.0008$ & $\$ 0.0056$ \\
\hline Annual minutes per line & & & 5595.47 \\
\hline Monthly minutes per line & & & 466.29 \\
\hline Daily minutes per line & & & 15.33 \\
\hline
\end{tabular}

Table 2

Table 2 can be interpreted for application to TELRIC costs as well by reading the results directly (as opposed to incrementally in comparison with Table 1). That is, the TELRIC notion incorporates the average cost of the elements used for the interconnection service, with the averaging taking place over all usage of those elements, rather than only over the interconnection. Thus, TELRIC for interconnection in this example is 0.56 cents per minute of traffic, or alternatively $\$ 24.81$ per line per year plus 1.1 cents per minute of traffic, or alternatively, $\$ 88.10$ per ccs or $\$ 291.46$ per Erlang.

\section{Discussion and conclusion}

The notion of TELRIC has been attacked by various parties for various reasons. ILECs in the United States have claimed that the notion of independent cost estimation based on a "theoretical" network represents constitutional takings, an argument that both the Eighth Circuit Court of Appeals and the Supreme Court seem to have rejected. Others have opposed the use of TELRIC - or, for that matter, any cost-based approach - on philosophical grounds, essentially rejecting any notion of regulation. In this paper, we do not attempt to address either of these arguments (we are neither lawyers nor philosophers). However, what we believe is worth understanding is that the TELRIC standard is (a) feasible; and (b) does not represent an attempt to degrade the profitability of operators.

TELRIC is probably best understood as a tool useful in resolving the classical informational asymmetry problems between regulator and regulated (see Laffont (1994) ). Properly implemented, it can be helpful in reducing some of the welfare losses associated 
with the regulator's imperfect knowledge of the cost structure of the regulated industry. As the example shows, it can also be helpful in implementing a price discrimination regime within the regulatory structure which may lead to welfare gains. The modeling approach adds understanding to the pricing problem more generally as well. Traditionally, interconnection prices have in nearly all countries been denominated by minutes of use. Yet, as the model clearly illustrates, minutes of use do not drive costs at all (except during the peak hour), making any such pricing scheme inefficient. Using the simulation model, it is possible to derive a more rational pricing scheme that would be denominated by some measure of capacity, such as Erlangs (which could translate into $\mathrm{MHz}$ of bandwidth).

Some parties have argued that it is preferable to use accounting standards for many of the purposes for which TELRIC was developed. The usual argument put forth by such advocates is, for example, that "simpler rules such as accounting rules, however, are far more transparent to the world, are not easily corrupted, are easily appealed, and provide a greater degree of market certainty and stability". ${ }^{9}$ Recent accounting scandals in the United States together with the results of this paper suggest that in fact, if a regulator implements a TELRIC methodology with the appropriate level of transparency, the argument may in fact run in the opposite direction.

\section{$7 \quad$ References}

Bush, C.A., V. Gupta, D. M. Kennet, J. Prisbrey, and W.W. Sharkey (2001). "Computer Modeling of the Forward-Looking Economic Cost of Local Exchange Telephone Networks: An Optimization Approach" Telecommunication Systems 18:4, 359-383.

DeGraba, Patrick. (2000). "A General Theory of Interconnection," mimeo. Federal Communications Commission. Federal Communications Commission (1996) First Report and Order, FCC 96-325.

Furchtgott-Roth, Harold (1998), "Statement of Commissioner Harold Furchtgott-Roth Re: Federal-State Joint Board on Universal Service; Forward-Looking Mechanism for high Cost Support for Non-Rural LECs ; (CC Docket Nos. 96-45, 97-160)," U.S. Federal Communications Commission.

Gasmi, Farid, and D. Mark Kennet, Jean-Jacques Laffont, and William W. Sharkey (2001), Cost Proxy Models and Telecommunications: A New Empirical Approach to Regulation, MIT Press.

Laffont, J.-J. (1994), “The New Economics of Regulation Ten Years After," Econometrica 62, pp. 507-537.

Laffont, J.-J, and J. Tirole, (2000) "Competition in Telecommunications," MIT Press. Press, W. H., B.P. Flannery, S.A. Teukolsky, and W.T. Vetterling (1986). Numerical Recipes: The Art of Scientific Computing. Cambridge: Cambridge University Press.

\footnotetext{
${ }^{9}$ Furchtgott-Roth (1998).
} 
Verizon Communications et al. v. Federal Communications Commission et al. (2002), Nos. 00-511, 00-555, 00-587, 00-590, and 00-602. 